\title{
Forced vibrations of tanks partially filled with liquid under seismic load
}

\author{
V. Gnitko, U. Marchenko, V. Naumenko \& E. Strelnikova \\ Institute for Mechanical Engineering Problems of the Ukrainian \\ Academy of Sciences, Ukraine
}

\begin{abstract}
Cylindrical tanks partially filled with liquid are the most general type of reservoirs for oil and other chemical-dangerous agent storage. Destruction of such tanks under seismic or impulsive load can lead to negative ecological consequences. The analysis method of dynamic behavior of cylindrical tanks partially filled with liquid that are under short-time impulsive load is under consideration. The method relies on reducing the problem of determining the fluid pressure to the system of singular integral equations. The coupled problem is solved using combination BEM and FEM. Differential equations of transient problem are solved numerically by Runge-Kutta method of 4th and 5th order. Numerical investigations of forced vibrations of the cylindrical tank filled with the incompressible fluid under seismic load have been carried out.
\end{abstract}

Keywords: forced vibrations, fluid-structure interaction, seismic load, boundary and finite element methods.

\section{Introduction}

Practicing engineers face many issues and challenges on the design and seismic evaluation of liquid storage tanks [1-3]. Liquid storage tanks are important components of lifeline and industrial facilities. Ground-supported cylindrical tanks are used to store a variety of liquids: water for drinking and firefighting, crude oil, wine, liquefied natural gas (LNG), etc. Failure of tanks, following destructive earthquakes, may lead to environmental hazard, loss of valuable contents, and disruption of fire-fighting effort. Inadequately designed or detailed tanks have suffered extensive damage in past earthquakes and have resulted in disastrous effects. Earthquake damage to steel tanks can take several forms. 
Large axial compressive stresses due to beamlike bending of the tank wall can cause "elephant-foot" buckling of the wall. Sloshing liquid near the free surface can damage the roof and upper shell of tank. High stresses in the vicinity of poorly detailed base anchors can rupture the tank wall. Base shears can overcome friction causing the tank to slide.

The forces on the tanks during an earthquake depend on the dynamics of the structure, fluid stored in the tank and of the founding soil. For dynamic behavior characterization of the containers partially filled with liquid two levels of interactions need to be studied. First being the interaction between the structure and liquid stored in the container. The second interaction is that between the structure and the foundation soil. The assumption of the base of the tank being fixed is valid if it is founded on hard rock.

Procedures for the seismic analysis and design of storage tanks are generally based on the Housner multicomponent spring-mass analogy. The analogy allows the complex dynamic behaviour of a tank and its contents to be considered in simplified form. The principal modes of response include a short period impulsive mode, with a period of around 0.5 seconds or less, and a number of longer period convective (sloshing) modes with periods up to several seconds. For most tanks, it is the impulsive mode, which dominates the loading on the tank wall. The first convective mode is usually much less significant than the impulsive mode, and the higher order convective modes can be ignored.

The dynamic analysis of shell structures is often performed by use of finite element (FE) programs [4]. But, such 3-D nonlinear finite element analysis, including the contained fluid as well as the foundation soil in the system, is complex and extremely time consuming. Several simplified theoretical investigations were also conducted and a few of these studies have been used as a basis for current design standards. In [5-9] authors offer the approach based on using the boundary element method to the problem of natural vibrations of the fluid-filled elastic shells of revolution, as well as to the problem of natural liquid vibrations in the rigid vessels. This approach has the certain advantages. In the basic equations the functions and their derivatives will be defined on the domain boundaries only. That allows reducing the order of dimension of problem. This method gives new qualitative possibilities in modeling the dynamic coupled problem.

\section{Problem statement}

Let us consider the coupled problem of dynamic behavior of an elastic shell of revolution partially filled with liquid subjected to short-time impulsive load (Figure 1). Also free and forced vibrations of such elastic and rigid shells are under consideration.

In this study the contained liquid is assumed to be inviscid and incompressible resulting in an irrotational flow field. Let $V_{x} ; V_{y} ; V_{z}$ are the fluid 
velocity vector components, then the incompressibility condition can be written as following

$$
\operatorname{div} V=\frac{\partial V_{x}}{\partial x}+\frac{\partial V_{y}}{\partial y}+\frac{\partial V_{z}}{\partial z}=0 .
$$

Under these suppositions, there exists a velocity potential defined as

$$
V_{x}=\frac{\partial \varphi}{\partial x}, \quad V_{y}=\frac{\partial \varphi}{\partial y}, \quad V_{z}=\frac{\partial \varphi}{\partial z} .
$$

Due to (1) potential $\varphi$ satisfies the Laplace equation

$$
\frac{\partial^{2} \varphi}{\partial x^{2}}+\frac{\partial^{2} \varphi}{\partial y^{2}}+\frac{\partial^{2} \varphi}{\partial z^{2}}=0
$$

The operator form of governing equations of motion for the liquid-structure system subjected to impulsive loading is given by

$$
\boldsymbol{L} \boldsymbol{U}+\boldsymbol{M} \ddot{\boldsymbol{U}}=P_{l}+Q,
$$

where $\boldsymbol{L}, \boldsymbol{M}$ are operators of elastic and mass forces of the shell; $\boldsymbol{U}=\left(u_{1}, u_{2}, u_{3}\right)$ is the displacement vector; $Q(t)$ is the vector of external surface load, $P_{l}$ is hydrodynamic pressure. The hydrodynamic pressure, according to the CauchyLagrange integral, can be represented as follows

$$
\frac{P}{\rho_{l}}=-\frac{\partial \varphi}{\partial t}-g z+\frac{P_{0}}{\rho_{l}},
$$

where $\varphi$ is the velocity potential, $\rho_{l}$ is the fluid density, $z$ is coordinate of a point in liquid counted in vertical direction, $g$ is the gravitational acceleration.

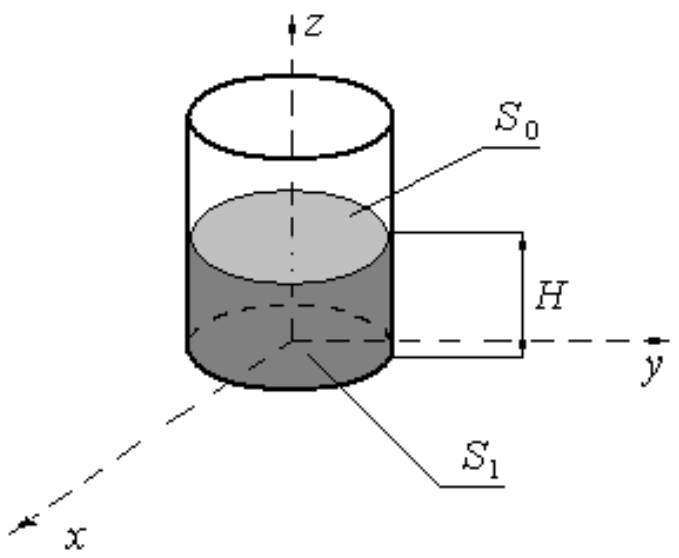

Figure 1: Cylindrical tank filled with liquid.

We denote a moistened surface of a shell through $S_{1}$ and a free surface as $S_{0}$ (Figure 1). Let refer the Cartesian coordinate system 0xyz connected with a shell. The free surface of the liquid $S_{0}$ coincides with the plane $x 0 y$ in unperturbed state. When a liquid storage tank is subjected to a dynamic load the boundary 
equations on the free surface are obtained by formulating dynamic and kinematics boundary conditions. The dynamic boundary condition is that the pressure on the free surface must be equal to the atmospheric one and the kinematics boundary condition is that liquid particles of the free surface remain on it during subsequent motion.

So we obtain the following boundary value problem

$$
\begin{gathered}
\boldsymbol{L} \boldsymbol{U}+\boldsymbol{M} \ddot{\boldsymbol{U}}+\rho_{l} \dot{\varphi}+g z=Q, \\
\frac{\partial \varphi}{\partial n}=\frac{\partial w}{\partial t}, P \in S_{1} ; \frac{\partial \varphi}{\partial n}=\dot{\zeta}, P \in S_{0} ; \dot{\varphi}+g \zeta=0, P \in S_{0}
\end{gathered}
$$

for defining the unknown functions $\boldsymbol{U}$ and $\varphi$.

\section{The mode superposition method for coupled dynamic problems}

We will seek the natural modes of shell vibration in the fluid in the following form

$$
U(x, y, z, t)=\sum_{k=1}^{m} u_{k}(x, y, z) c_{k}(t),
$$

where functions $u_{k}(x, y, z)$ are modes of natural vibrations in vacuum, $c_{k}(t)$ are unknown factors.

We will seek $\varphi$ as a sum of two potentials

$$
\varphi=\varphi_{1}+\varphi_{2} .
$$

To determine $\varphi_{1}$ we obtain the following boundary value problem:

$$
\nabla^{2} \varphi_{1}=0, \frac{\partial \varphi_{1}}{\partial n}=\frac{\partial w}{\partial t}, P \in S_{1}, \frac{\partial \varphi_{1}}{\partial t}=0, P \in S_{0} .
$$

Here $w(x, y, z, t)=\sum_{k=1}^{m} w_{k}(x, y, z) c_{k}(t)$, and functions $w_{k}(x, y, z)$ are normal modes of natural vibrations in vacuum.

It would be noted, that from equation (3) and second one from (5) follows

$$
\varphi_{1}(x, y, z, t)=\sum_{k=1}^{m} \varphi_{1 k}(x, y, z) \dot{c}_{k}(t) .
$$

To determine $\varphi_{1 k}$ we have the following boundary value problem:

$$
\begin{gathered}
\nabla^{2} \varphi_{1 k}=0, \\
\frac{\partial \varphi_{1 k}}{\partial n}=w_{k}, P \in S_{1}, \\
\varphi_{1 k}=0, P \in S_{0} .
\end{gathered}
$$

To determine $\varphi_{2}$ we will have the following relation

$$
\varphi_{2}(x, y, z, t)=\sum_{k=1}^{n} \varphi_{2 k}(x, y, z) d_{k}(t),
$$


where functions $\varphi_{2 k}$ are the natural modes of fluid vibration in rigid vessel. Let us formulate the problem of fluid vibration in rigid vessel relative to some function $\varphi_{r}$

$$
\begin{gathered}
\nabla^{2} \varphi_{r}=0, \\
\frac{\partial \varphi_{r}}{\partial n}=0, P \in S_{1}, \\
\frac{\partial \varphi_{r}}{\partial n}=\dot{\zeta}, P \in S_{0}, \\
\dot{\varphi}_{r}+g \zeta=0, P \in S_{0} .
\end{gathered}
$$

The last equation (8) follows from equation (3) and it is the dynamic condition on free surface. Differentiating this equation with respect to $t$ we come to the following equation of fluid vibrations in the rigid vessel:

$$
\ddot{\varphi}_{r}+g \frac{\partial \varphi_{r}}{\partial n}=0, P \in S_{0} .
$$

Let us seek for the solution of mentioned problem in the next form

$$
\varphi_{r}(x, y, z, t)=e^{i \kappa t} \psi(x, y, z) .
$$

For function $\psi$ we will have the following problem of free harmonic fluid vibrations

$$
\begin{gathered}
\nabla^{2} \psi=0, \\
\frac{\partial \psi}{\partial n}=0, P \in S_{1}, \\
\frac{\partial \psi}{\partial n}=\frac{\kappa^{2}}{g} \psi, P \in S_{0} .
\end{gathered}
$$

Solving of this problem one can obtain the number of eigenvalues $\kappa_{\mathrm{k}}$ and corresponding natural modes, namely functions $\psi_{k}$. After equation (10) is solved we are looking for function $\varphi_{2}$ in the form

$$
\begin{gathered}
\varphi_{2}(x, y, z, t)=\sum_{k=1}^{n} d_{k}(t) \psi_{k}(x, y, z) ; \\
\varphi_{2 k}(x, y, z)=\psi_{k}(x, y, z)
\end{gathered}
$$

So we have $\varphi=\varphi_{1}+\varphi_{2}$, where

$$
\varphi_{1}(x, y, z, t)=\sum_{k=1}^{m} \varphi_{1 k}(x, y, z) \dot{c}_{k}(t), \quad \varphi_{2}(x, y, z, t)=\sum_{k=1}^{n} d_{k}(t) \psi_{k}(x, y, z) .
$$

then

$$
\begin{gathered}
\nabla^{2} \varphi=\nabla^{2} \varphi_{1}+\nabla^{2} \varphi_{2}=0, \\
\frac{\partial \varphi}{\partial n}=\frac{\partial \varphi_{1}}{\partial n}+\frac{\partial \varphi_{2}}{\partial n}=\frac{\partial w}{\partial t}, P \in S_{1} .
\end{gathered}
$$

On free surface it is required

$$
\frac{\partial \varphi}{\partial n}=\dot{\zeta}, P \in S_{0} ; \dot{\varphi}+g \zeta=0, P \in S_{0} .
$$


Differentiating last equation with respect to $t$ we come to the equation

$$
\ddot{\varphi}+g \frac{\partial \varphi}{\partial n}=0, P \in S_{0} .
$$

We have $\dot{\varphi}_{1}=\ddot{\varphi}_{1}=0$ from relation (7). Therefore (12) leads to the system of differential equations

$$
\sum_{k=1}^{n} \ddot{d}_{k}(t) \psi_{k}(x, y, z)+g \sum_{k=1}^{m} \dot{c}_{k}(t) \frac{\partial \varphi_{1 k}(x, y, z)}{\partial n}+g \sum_{k=1}^{n} d_{k}(t) \frac{\partial \psi_{k}(x, y, z)}{\partial n}=0 .
$$

Using the relations

$$
\frac{\partial \psi_{k}}{\partial n}=\frac{\kappa_{k}^{2}}{g} \psi_{k}, P \in S_{0}
$$

we obtain

$$
\sum_{k=1}^{n}\left[\ddot{d}_{k}(t)+\kappa_{k}^{2} d_{k}(t)\right] \psi_{k}(x, y, z)+g \sum_{k=1}^{m} \dot{c}_{k}(t) \frac{\partial \varphi_{1 k}(x, y, z)}{\partial n}=0 .
$$

Due to orthogonality of natural modes of fluid vibrations in rigid vessel we have after dot product of equation (12) by functions $\psi_{l}$

$$
\ddot{d}_{l}(t)+\kappa_{l}^{2} d_{l}(t)+\frac{g}{\left(\psi_{l}, \psi_{l}\right)} \sum_{k=1}^{m} \dot{c}_{k}(t)\left(\frac{\partial \varphi_{1 k}}{\partial n}, \psi_{l}\right)=0, \quad l=1,2 . ., n .
$$

When functions $\varphi_{k}$ and $\psi_{k}$ are obtained we substitute them in equation (2) and obtain the following equation

$$
\boldsymbol{L}\left(\sum_{k=1}^{m} c_{k} u_{k}\right)+\boldsymbol{M}\left(\sum_{k=1}^{m} \ddot{c}_{k} u_{k}\right)=-\rho_{l}\left(\sum_{k=1}^{m} \ddot{c}_{k} \varphi_{1 k}+\sum_{i=1}^{n} \dot{d}_{i} \psi_{i}+g z\right)+Q .
$$

Let us $\omega_{k}, u_{k}$ are natural frequencies and free vibrations modes of the shell in vacuum. Then the following relationships are valid

$$
\boldsymbol{L} u_{k}=\omega_{k}^{2} \boldsymbol{M} u_{k}, \quad\left(\boldsymbol{M} u_{k}, u_{j}\right)=\delta_{k j} .
$$

Considering the result of dot product of equation (15) by $u_{j}$ and taking into account relationships (14) and (16), we come to the next set of $n+m$ second order differential equations

$$
\begin{gathered}
\ddot{c}_{j}(t)+\omega_{j}^{2} c_{j}(t)+\rho_{L} \sum_{k=1}^{m} \ddot{c}_{k}\left(\varphi_{k}, u_{j}\right)+\sum_{i=1}^{n} \dot{d}_{i}\left(\psi_{i}, u_{j}\right)+g\left(z, u_{j}\right)=\left(Q, u_{j}\right), j=1, m \\
\ddot{d}_{l}(t)+\kappa_{l}^{2} d_{l}(t)+\frac{g}{\left(\psi_{l}, \psi_{l}\right)} \sum_{k=1}^{m} \dot{c}_{k}(t)\left(\frac{\partial \varphi_{1 k}}{\partial n}, \psi_{l}\right)=0, \quad l=1,2 . ., n
\end{gathered}
$$

So we reduce the considered problem to the following. First, we have to obtain the natural frequencies and free vibrations mode shapes of the elastic shell in vacuum. The problem is solved using FEM. Second, it is necessary to obtain the frequencies and free vibrations modes of liquid in rigid shell under force of gravity. Then we define the frequencies and free vibrations modes of elastic shell without including the force of gravity. These two problems are solved using 
BEM. And at the end we solve the set of second order differential equations using 4th and 5th order Runge-Kutta method.

\section{System of the boundary integral equations}

We use furthermore the cylindrical coordinate system and represent unknown functions as Fourier series by circumferential coordinate

$$
w=w(r, z) \cos \alpha \theta, \quad \varphi=\varphi(r, z) \cos \alpha \theta .
$$

To solve the coupled hydro-elasticity problem it is necessary to determine the potentials $\varphi_{1}$ and $\varphi_{2}$.

These problems here are reduced to the solution of the systems of singular integral equations. Determination of the potentials $\varphi_{1}$ was accomplished as in $[10,11]$. So we obtain the following system

$$
\begin{gathered}
2 \pi \varphi\left(z_{0}\right)+\int_{\Gamma} \varphi(z) \Theta\left(z, z_{0}\right) r(z) d \Gamma-\int_{0}^{R} q(\rho) \Phi\left(P, P_{0}\right) \rho d \rho= \\
=\int_{\Gamma} w(z) \Phi\left(P, P_{0}\right) r(z) d \Gamma_{1}, \quad P_{0} \in S_{1}, \\
\int_{\Gamma} \varphi(z) \Theta\left(z, z_{0}\right) r(z) d \Gamma-\int_{0}^{R} q(\rho) \Phi\left(P, P_{0}\right) \rho d \rho=\int_{\Gamma} w(z) \Phi\left(P, P_{0}\right) r(z) d \Gamma_{1}, P_{0} \in S_{0},
\end{gathered}
$$

where

$$
\begin{gathered}
\Theta\left(z, z_{0}\right)=\frac{4}{\sqrt{a+b}}\left\{\frac{1}{2 r}\left[\frac{r^{2}-r_{0}^{2}+\left(z_{0}-z\right)^{2}}{a-b} \mathrm{E}_{\alpha}(k)-\mathrm{F}_{\alpha}(k)\right] n_{r}+\frac{z_{0}-z}{a-b} \mathrm{E}_{\alpha}(k) n_{z}\right\}, \\
\Phi\left(P, P_{0}\right)=\frac{4}{\sqrt{a+b}} \mathrm{~F}_{\alpha}(k) .
\end{gathered}
$$

Here the following notations are introduced

$$
\begin{gathered}
\mathrm{E}_{\alpha}(k)=(-1)^{\alpha}\left(1-4 \alpha^{2}\right)^{\pi / 2} \cos 2 \alpha \theta \sqrt{1-k^{2} \sin ^{2} \theta} d \theta, \\
\mathrm{F}_{\alpha}(k)=(-1)^{\alpha} \int_{0}^{\pi / 2} \frac{\cos 2 \alpha \theta d \theta}{\sqrt{1-k^{2} \sin ^{2} \theta}}, \quad k^{2}=\frac{2 b}{a+b}
\end{gathered}
$$

Letting $\alpha=0$ in the above expressions, we obtain the standard elliptic first and second kind integrals.

To determine the potential $\varphi_{2}$ we have to obtain functions $\psi_{k}$. Let us denote by $\psi_{1 k}$ the values of $\psi_{k}$ on the wetted surface $S_{1}$ and by $\psi_{0 k}$ the values of $\psi_{k}$ on the free surface $S_{0}$. Using the direct formulation of BEM to solve boundary value problem (10) and skipping for convenience an index $k$ we can write the following system of singular integral equations 


$$
\begin{gathered}
2 \pi \psi_{1}+\iint_{S_{1}} \psi_{1} \frac{\partial}{\partial n}\left(\frac{1}{r}\right) d S_{1}-\frac{\kappa^{2}}{g} \iint_{S_{0}} \psi_{0} \frac{1}{r} d S_{0}+\iint_{S_{0}} \psi_{0} \frac{\partial}{\partial z}\left(\frac{1}{r}\right) d S_{0}=0 \\
-\iint_{S_{1}} \psi_{1} \frac{\partial}{\partial n}\left(\frac{1}{r}\right) d S_{1}-2 \pi \psi_{0}+\frac{\kappa^{2}}{g} \iint_{S_{0}} \psi_{0} \frac{1}{r} d S_{0}=0
\end{gathered}
$$

Suppose that

$$
\psi=\psi(r, z) \cos \alpha \theta
$$

we obtain for each harmonic term the following system of singular integral equations

$$
\begin{gathered}
\iint_{S_{1}} \psi \frac{\partial}{\partial n}\left(\frac{1}{r\left(P, P_{0}\right)}\right) d S_{1}=\int_{r} \psi(z) \Theta\left(z, z_{0}\right) r(z) d \Gamma ; \\
\iint_{S_{0}} \psi\left(\frac{1}{r\left(P, P_{0}\right)}\right) d S_{0}=\int_{0}^{R} \psi(\rho) \Phi\left(P, P_{0}\right) \rho d \rho .
\end{gathered}
$$

Here kernels $\Theta\left(z . z_{0}\right)$ and $\Phi\left(P, P_{0}\right)$ are defined in (20).

For numerical solution of system described by equations (19), (22) the boundary element method with constant approximation of unknown density on elements was used.

\section{Numerical results}

It would be noted that in the dynamic problem of horizontal seism we have to consider only $\alpha=1$ in expressions (18), (21) because only first circumferential mode is excited by seismic motion.

To validate the developed numerical algorithm the comparison with FEM results was accomplished. Let us consider a cylindrical shell with a flat bottom partially filled with the fluid. The geometry of the tank is shown in Figure 2 and the parameters are following: the radius is $R=1 \mathrm{~m}$, the thickness is $h=0.01 \mathrm{~m}$, the length $L=2 \mathrm{~m}$, Young's modulus $E=2 \cdot 10^{5} \mathrm{MPa}$, Poisson's ratio $v=0.3$, the material's density is $\rho=7800 \mathrm{~kg} / \mathrm{m}^{3}$, the fluid density $\rho_{\mathrm{l}}=1000 \mathrm{~kg} / \mathrm{m}^{3}$. The filling level of the fluid is denoted as H. Boundary conditions are following: $u_{r}=u_{z}=u_{\theta}=0$ to $z=0$ and $r=R$.

We analyze forced vibrations coupled problem. The radial load (Figure 3 ) is suddenly applied to cylindrical surface of the tank $q(r, z, t)=q_{0} \cos k \varphi(r, z) \exp (-t / \tau)$, where $q_{0}=0.1 \mathrm{MPa}, \tau=14.2 \cdot 10^{-6} \mathrm{~s}$. Time at the end $t_{n}=5 \cdot 10^{-3} \mathrm{~s}$.

The radial displacement response was calculated in four points that are shown in Figure 3, point 1 (node 91) is situated in the wetted part of the wall, point 2 (node 121) belongs to boundary of the liquid free surface, point 3 (node 69) is almost on the bottom whereas point 4 (node 161) is on the top of the wall. Figures 5-8 are representative of the forced motion response as calculated by the proposed method - solid lines and by the finite element complex - dash lines. 


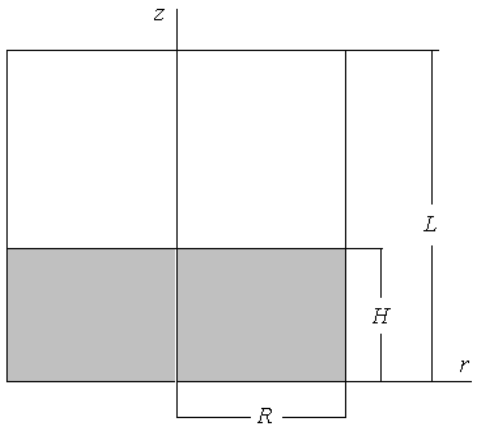

Figure 2: The scheme of the cylindrical tank.

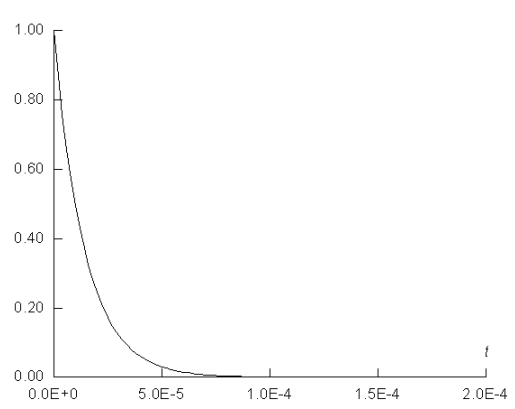

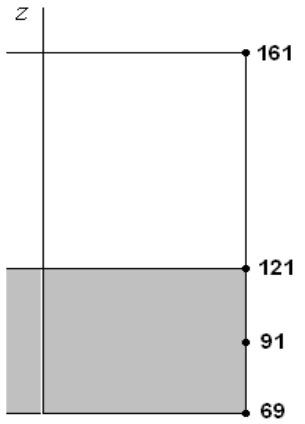

Figure 3: The scheme of nodes.

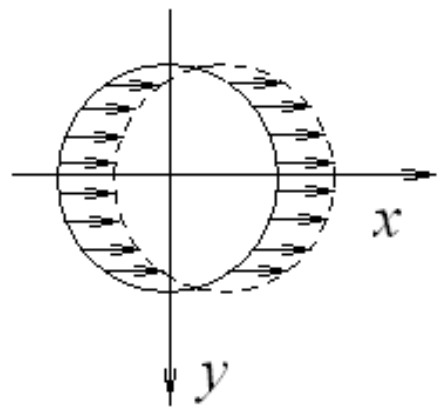

Figure 4: Impulsive load.

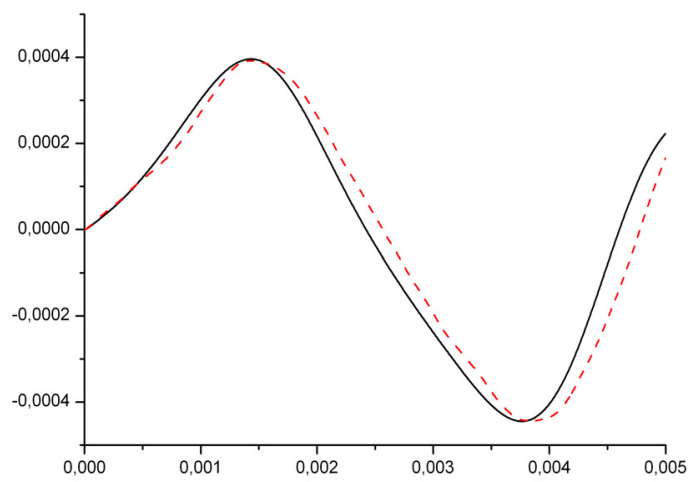

Figure 5: $\quad$ Time history of the radial displacement at the point 1 . 


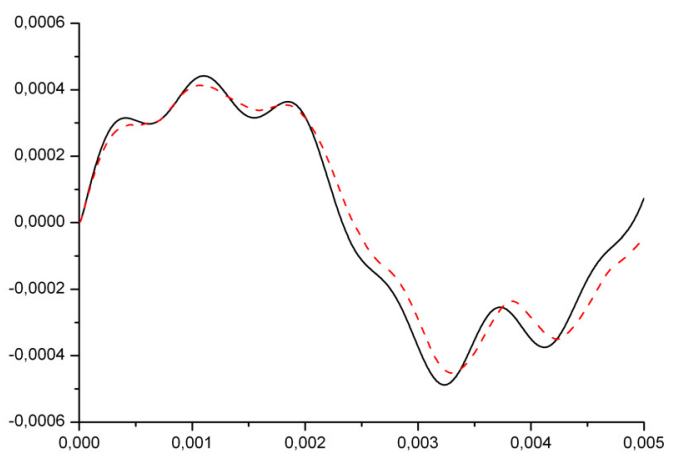

Figure 6: Time history of the radial displacement at the point 2.

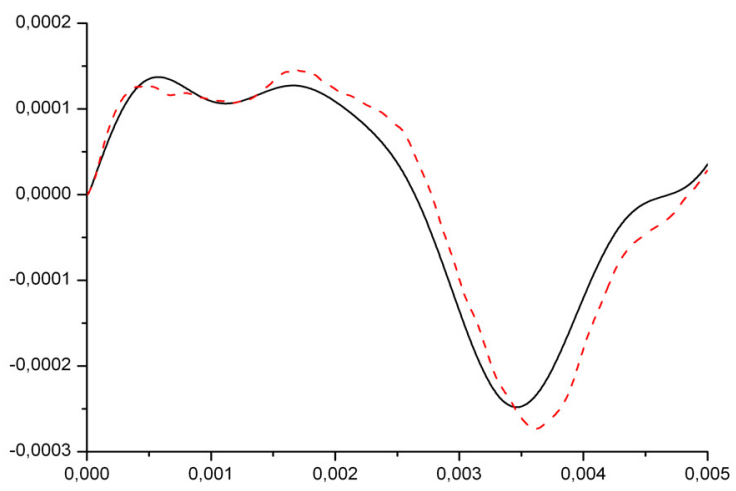

Figure 7: Time history of the radial displacement at the point 3.

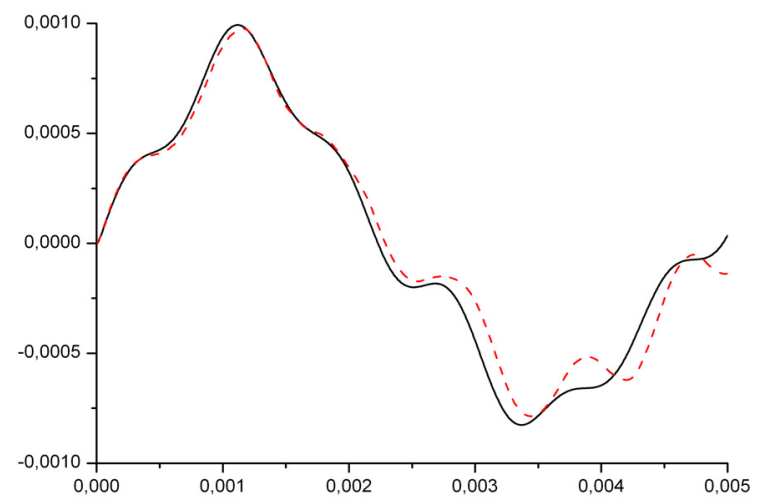

Figure 8: $\quad$ Time history of the radial displacement at the point 4 . 
The figures demonstrate good agreement of results obtained by different methods. That testifies the reliability of the method and algorithm proposed.

From previous investigation we found out that maximal radial displacement was in the point on the boundary between water and air (further "control point"). It will be interesting how the radial displacement at the control point will change subject to various filling levels of the fluid. So Figure 9 demonstrates the time history of the radial displacement at the control point when filling level of the fluid $\mathrm{H}=40 \mathrm{~cm}, \mathrm{H}=80 \mathrm{~cm}, \mathrm{H}=190 \mathrm{~cm}$.

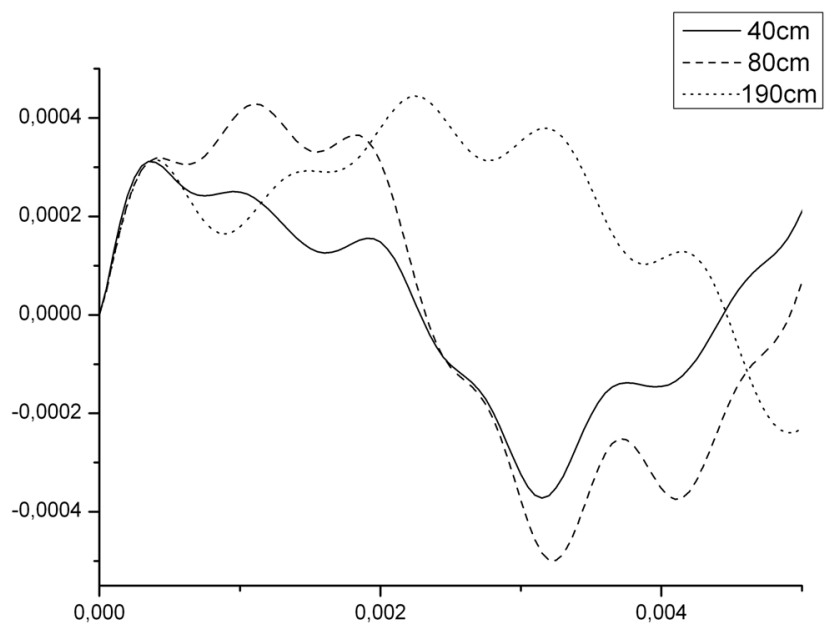

Figure 9: Time history of the radial displacement at the control point.

\section{Conclusions}

The numerical procedure based on a coupling the finite element formulation and the boundary element method is developed for the forced vibration analysis of shells of revolution with an arbitrary meridian partially filled with the fluid. We introduce the representation of the velocity potential as the sum of two potential corresponding to problem of the fluid free vibrations in the rigid shell and the one corresponding to problem of elastic shell with fluid without including the gravitational component. Integration by the fluid volume is reduced to integrals along the shell meridian and along the radius of the liquid free surface. It is the basic advantage of our method based on a combination of the boundary integral equations method, finite element method and expansion into Fourier series. The governing integral equations for each harmonic have been obtained. The forced vibration problem includes the liquid added masses into equations of motion. Numerical investigations of natural frequencies and forced vibrations of the cylindrical tank filled with the incompressible fluid have been carried out. 


\section{Acknowledgements}

The authors gratefully acknowledge the sponsorship of this research by the Science and Technology Center in Ukraine in framework of the Project \#4624. Authors also would like to acknowledge our STCU Project collaborators Professors Carlos Brebbia and Eduard Ventsel for their constant support and interest in our research.

\section{References}

[1] Sanchez-Sanchez, H., Cortes, S.C., Dominguez, A.M., Structural behaviour of liquid filled storage tanks of large capacity placed in seismic zones of high risk in Mexico// Proc. of $13^{\text {th }}$ World Conference on Earthquake Engineering, Vancouver, B.C., Canada, 2004, Paper No. 2665.

[2] Sanchez-Sanchez, H., Cortes, S.C., Seismic response of cylindrical tanks for oil// Proc. of $14^{\text {th }}$ World Conference on Earthquake Engineering, Beijing, China, 2008.

[3] Jhung, M.J., Jo, J.C., Jeong, S.J., Impact analysis of a water storage tank, Nuclear Engineering and Technology, 38(7), 2006.

[4] Kubenko, V.D. \& Koval'chuk, P.S., Nonlinear problems of the dynamics of elastic shells partially filled with a liquid. International Journal of Applied Mechanics, 36(4), pp. 421-448, 2000.

[5] Amabili, M. \& Païdoussis, M. P., Review of studies on geometrically nonlinear vibrations and dynamics of circular cylindrical shells and panels, with and without fluid-structure interaction. Applied Mechanics Review, 56(4), pp. 349-381, 2003.

[6] Kumar, V. \& Ganesan, N., Dynamic analysis of conical shells conveying fluid. Journal of Sound and Vibration, 310(1-2), pp. 38-57, 2008.

[7] Malhotra, P. K., New method for seismic isolation of liquid-storage tanks. Journal of Earthquake Engineering and Structural Dynamics, 26(8), pp. 839-847, 1997.

[8] Zhang, Y.L., Gorman, D.G. \& Reese, J.M., Vibration of prestressed thin cylindrical shells conveying fluid. Thin-Walled Structures, 41, pp. 1103-1127, 2003.

[9] Chen, Y.H., Hwang, W.S. \& Ko, C.H., Numerical simulation of the threedimensional sloshing problem by boundary element method. Journal of the Chinese Institute of Engineers, 23(3), pp. 321-330, 2000.

[10] Brebbia, C.A., Telles, J.C.F. \& Wrobel, L.C. Boundary Element Techniques, Springer-Verlag: Berlin and New York, 1984.

[11] Strelnikova E.,Yeseleva E., Gnitko V., Naumenko V. Free and forced vibrations of the shells of revolution interacting with the liquid// Proc. of XXXII Conference "Boundary elements and other mesh reduction methods” WIT Press, Transaction on Modeling and Simulation, 2010, P. 203-211. 